\title{
Microanalysis: What Is It, Where Did It Come From, and Where Is It Going?
}

\author{
Dale E. Newbury \\ National Institute of Standards and Technology, Gaithersburg, MD 20899-8370
}

"Microanalysis" in the parlance of the Microanalysis Society (MAS) refers to spatially-resolved elemental and molecular analysis performed at the micrometer to nanometer to picometer scales by any combination of excitation and analytical spectrometry that yields useful information. MAS evolved from the Electron Probe Analysis Society of America (EPASA), founded in 1966 because of intense interest in electron-excited X-ray microanalysis (EPMA) with wavelength dispersive spectrometry (WDS), the first successful elemental analysis technique with micrometer resolution [1]. EPMA's birth came in the extraordinary $1951 \mathrm{Ph}$. D. thesis of Raymond Castaing, who as a graduate student not only built the first successful electron microprobe with X-ray spectrometry and Kossel X-ray diffraction capabilities but also described the physical basis for converting the measured X-ray intensities into concentration values [2]. In the 1950s Castaing's continuing work with his colleagues and students to improve all aspects of the EPMA technique soon attracted international scientific interest as well as that of instrument manufacturers. A "zero-eth" U.S. meeting held at the U.S. Naval Research Laboratory in 1958 brought together twenty international participants to discuss the rapidly emerging method. 1958 also marked the appearance of the first commercial electron probe microanalyzer by Cameca. With 15 years of international activity by the time of the first EPASA conference in 1966 (four international congresses on X-ray optics and microanalysis had already been held), the remarkable program of 53 presentations is striking for the range of topics and sophistication. A partial selection from the conference program reveals:

Andersen: The Quality of X-ray Microanalysis in the Ultra-Soft X-ray Region

Beaman: The Evaluation of the Correction Procedures Used in EPMA for $Z=12$ to 33 .

Borovskii: The Theory of Quantitative Local X-ray Spectral Analysis

Brown: Study of Secondary Fluorescence Using a Sandwich Sample Technique

Colby: Microprobe Analysis by Means of Target Current Measurements

Duncumb: A Simple Correction Procedure for Ultra-Soft X-ray Microanalysis

Goldstein: Experimental Procedure for Trace Determination with the Electron Probe

Heinrich: Quantitative Electron Probe Microanalysis: Absorption Correction Uncertainty

Kimoto: "Quantitative Analysis of Light Elements with an EPMA

Lifshin: An Automated Method for the Collection and Analysis of Microbeam Probe Data Moll: Analytical Problems Associated with the Quantitative Determination of B, C, N, O Morris: Crystal Orientation and Lattice Constants from Kossel Lines

Ogilvie: Empirical Method for the Analysis of Mixed Oxide Phases

Openshaw: Modification of an EPMA for Analysis of Na to B

Rappaport: Increased Spatial Resolution of the Electron Probe

Rucklidge: A Computer Program for Processing Microprobe Data

Tousimis: Enzyme and Polysaccharide Localization in Biological Tissues with the EPMA

Trombka: Analytic Method for a Non-Dispersive analysis

Wittry: The Voltage Dependence of Cathodoluminescence in Semiconductors

Wolf: A Proposed Method for Calculating the Atomic Number Correction in EPMA

Ziebold: Empirical Calibrations for Metal Oxides and Carbides

If this list of topics seems familiar, consulting any recent MAS conference proceedings reveals that many are still the subjects of research 50 years later. Hopefully we are making some progress! 
And indeed we are! Thanks to the efforts of scientists and engineers in so many fields, today's manufacturers produce instrumentation with performance that our colleagues in 1966 could scarcely imagine. All elements except $\mathrm{H}$ and $\mathrm{He}$, which do not produce X-rays, can now be measured with a high degree of accuracy and precision as well as at trace sensitivity and at greatly improved spatial resolution. Moreover, problems that recently were considered solvable only with EPMA and high-spectral resolution WDS can now be solved with scanning electron microscopy and energy dispersive X-ray spectrometry (SEM/EDS), even for situations of severe peak interference and large elemental concentration ratios, e.g., quantitatively measuring minor and trace constituents when major constituent interference occurs [3, 4].

EPASA evolved into the Microbeam Analysis Society (MAS) and then into the Microanalysis Society to reflect the expansion of the microanalysis field beyond the electron beam excitation and $\mathrm{X}$-ray spectrometry of EPMA to include all analytical microscopies with electron, ion, photon, and electrical field excitation and with electron, ion, and photon spectrometry. Nearly every possible combination of excitation and spectrometry has been explored and likely presented at an MAS conference, with some techniques such as secondary ion mass spectrometry (note the important contribution of the secondary ion microscope by Castaing and Slodzian [5]!) developing into their own distinct conferences and scientific societies with early support from MAS.

An emerging theme that is likely to shape the near future of microanalysis is the rapid development of instrumentation capable of producing enormous streams of data. As computer control of instrument operation and of data collection has become ever more efficient and extensive, the resulting image and spectrometry data structures collected in a single user-mediated session, or indeed under autonomous computer control for extended periods, can overwhelm simple manual inspection. Moving forward, a major theme will be the development of software tools that can aid the analyst by efficiently mining the enormous data haystacks for the unexpected needles. As powerful and useful as these software tools have already become, and as likely as they are to be become indispensable as they continue to improve, the analyst must not degenerate into the unquestioning slave of the computer display. Careful analysis that can reach robust and defensible conclusions will always require the intellectual input of the analyst at every stage of the analytical process, from selecting and preparing the specimen, optimizing the selection of analytical conditions, choosing the meaningful locations to study, recognizing and pursuing the unexpected, and finally to interpreting the results. Experience, intuition, common sense as well as a sense of responsibility for the results are provided by the analyst, and ultimately the analyst not the computer software owns the result!

Disclaimer: Certain commercial equipment, instruments, or materials are identified in this paper to foster understanding. Such identification does not imply recommendation or endorsement by the National Institute of Standards and Technology, nor does it imply that the materials or equipment identified are necessarily the best available for the purpose.

References

[1] L. Marton and A. Tousimis, First National Conference on Electron Probe Microanalysis, May 4 -6, 1966, University of Maryland, College Park.

[2] R. Castaing "Application of Electron Probes to Local Chemical and Crystallographic Analysis" thesis, Univ. Paris, 1951 [translation by P Duwez and D. Wittry available at the MAS website: http://www.microbeamanalysis.org/history/Castaing-Thesis-clearscan.pdf)

[3] D. Newbury and N. Ritchie, J. Mats. Sci., 50 (2015) 493.

[4] D. Newbury and N. Ritchie, Micros. Microanal., 22 (2016) 520.

[5] R. Castaing and G. Slodzian, J. Microscopie, 1 (1962) 395. 